\title{
Marine Drugs Research in Saudi Arabia
}

\author{
Ahmed E Fazary* \\ Chemistry Department, Faculty of Science, King Khalid University, Kingdom of Saudi Arabia \\ Applied Research Sector, Egyptian Organization for Biological Products and Vaccines (VACSERA Holding Company), Egypt
}

Received: November 16, 2017; Published: November 29, 2017

*Corresponding author: Ahmed E Fazary, Chemistry Department, Faculty of Science, King Khalid University, Abha 9004, Kingdom of Saudi Arabia; Applied Research Sector, Egyptian Organization for Biological Products and Vaccines (VACSERA Holding Company), 51 Wezaret El-Zeraa St, Agouza, Giza, Egypt; Tel: 966-7-241-8343; E-mails: aefazary@gmail.com; afazary@kku.edu.sa

\section{Opinion}

Recently drug discovery and development program based on the sustainable use of marine biodiversity have attracted much attention, because many scientists believe that its integration with recent advances in biotechnology not only promises economic benefits but also promotes the protection and conservation of marine biodiversity. Some biotechnological innovations have enabled to generate ecologically and environmentally sound approaches, which contribute greatly to the sustainable use of marine biodiversity. This emerging multidiscipline is especially interesting to be developed in Saudi Arabia as a wide country with highly diverse marine resources. Therefore here we will deal with developing a concept about how drug discovery based on marine natural products can be implemented to promote the sustainable use, protection and conservation of marine biodiversity as well as to secure economic benefits. Marine Organisms such as sponges and corals are broadly known as rich sources of novel and useful bioactive marine natural products as marine cancer drugs. However, it is not only elaborate pharmaceutically useful compounds but also produce a lot of toxic substances.

One of the most important societal contributions of applied chemists has been the isolation and identification of toxins responsible for seafood poisoning. Outbreaks of seafood poisoning are usually sporadic and unpredictable because toxic fish or shellfish do not produce the toxins themselves, but concentrate them from organisms that they eat. Most marine toxins are produced by microorganisms such as dinoflagellates or marine bacteria and may pass through several levels of the food chain. The identification of marine toxins has been one of the most challenging areas of marine natural products chemistry. The major occupation of marine natural products chemists for the past two decades has been the search for potential pharmaceuticals. It is difficult to single out a particular bioactive molecule that is destined to find a place in medicine. However, many compounds have shown promise. Marine organisms produce some of the most cytotoxic compounds ever discovered, but the yields of these compounds are invariably so small that natural sources are unlikely to provide enough material for drug development studies. These organisms are frequently colonized by bacteria. Some of these bacteria can be pathogenic or serve as beneficial symbionts. Therefore, these organisms need to regulate the bacteria they encounter and resist microbial pathogens.

Many of the natural products isolated from marine invertebrates share structural homology with compounds of microbial origin, leading to the hypothesis that the marine compounds are actually produced by microorganisms living in association with the invertebrates. Marine invertebrates are indeed largely sessile, filter-feeding organisms that contain a complex assemblage of symbiotic microorganism. Thus, the real producer of biologically active compounds from marine invertebrates is always uncertain. Detailed investigation of bioactive metabolite symbioses is a field that is still in its infancy. Whilst marine compounds are considered in current pharmacopoeia, it is anticipated that the aquatic environment will become an invaluable source of novel bioactive compounds in the nearest future. It was known that many of these natural products act as regulators of specific biological functions. Some of them have pharmacological activity due to their specific interactions with receptors and enzymes.

The development of marine compounds as therapeutic agents is still in its early stage due to the lack of an analogous ethno-medical history as compared with terrestrial habitats, together with the relative technical difficulties in collecting marine organisms. The systematic investigation of marine environments is reflected in the large number of novel compounds reported in the literature over the past decade. Some of these agents have entered preclinical and clinical trials, and it may be expected that this number will increase in the future. It was known that the isolation of new anticancer agents derived from marine sources has been based on the collection of marine macroorganisms. The progress in scuba-diving techniques and deep-water collection instruments has been pivotal in the collection programs implemented by academic and pharmaceutical groups. Recently, a lot of research programs are emerging to exploit marine microorganisms and the results are promising. These studies 
have demonstrated the capability of marine bacteria to produce compounds not available from terrestrial sources. They also have led to an increase in knowledge of the many bioactive compounds produced by these microorganisms. Nowadays, the compounds are systematically tested for relevant biomedical properties including antiproliferative effects. The major screening system is carried out by the National Cancer Institute of the USA. This system looks for selective activity in a panel of more than 60 human tumor cell lines. Alternative strategies employ a more mechanistic-based approach, with systems designed to screen for substances with inhibitory properties towards specific enzymatic reactions. This type of assay offers specificity and can focus on a number of discrete drug targets. The potentially confounding effects of toxic components are also avoided, permitting the screening of crude extracts from marine organisms. Thus, the search for new and useful bioactive natural products from marine soft corals and sponges has been one of the main research subjects and that research will be performed within several projects funded by King Abdulaziz City for Science and Technology in Riyadh, Saudi Arabia, and by many Saudi Arabia universities.

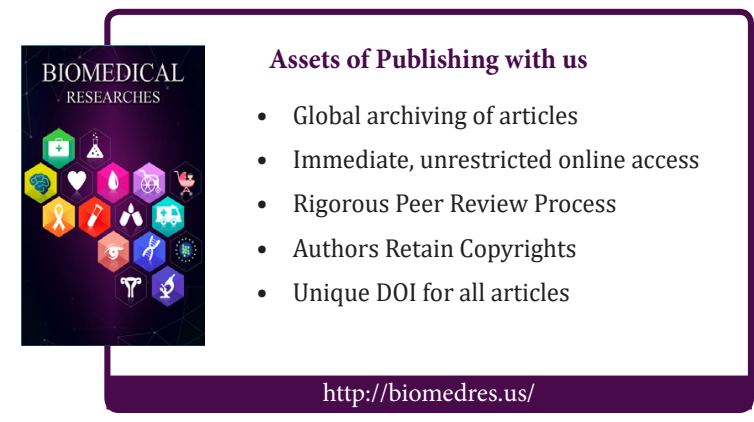

